\title{
Association Between a Variable Number Tandem Repeat Polymorphism Within the DAT1 Gene and the Mesolimbic Pathway in Parkinson's Disease
}

\author{
Stefan du Plessis ${ }^{1,2 *}$, Minke Bekker ${ }^{3}$, Chanelle Buckle ${ }^{1}$, Matthijs Vink ${ }^{4}$, Soraya Seedat ${ }^{1,2}$, \\ Soraya Bardien ${ }^{2,3}$, Jonathan Carr ${ }^{2,5}$ and Shameemah Abrahams ${ }^{2,3}$ \\ ${ }^{1}$ Department of Psychiatry, Faculty of Medicine and Health Sciences, Stellenbosch University, Cape Town, South Africa, \\ ${ }^{2}$ Stellenbosch University Genomics of Brain Disorders Research Unit, South African Medical Research Council, Cape Town, \\ South Africa, ${ }^{3}$ Division of Molecular Biology and Human Genetics, Faculty of Medicine and Health Sciences, Stellenbosch \\ University, Cape Town, South Africa, ${ }^{4}$ Departments of Developmental and Experimental Psychology, Utrecht University, \\ Utrecht, Netherlands, ${ }^{5}$ Division of Neurology, Faculty of Medicine and Health Sciences, Stellenbosch University, Cape Town, \\ South Africa
}

OPEN ACCESS

Edited by:

Andrew Anthony Hicks,

Eurac Research, Italy

Reviewed by:

Owen A. Ross,

Mayo Clinic, United States

Manabu Funayama

Juntendo University, Japan

Norbert Brüggemann,

University of Lübeck, Germany

*Correspondence:

Stefan du Plessis

stefandup@sun.ac.za

Specialty section:

This article was submitted to

Neurogenetics,

a section of the journal

Frontiers in Neurology

Received: 31 January 2020

Accepted: 28 July 2020

Published: 02 September 2020

Citation:

du Plessis S, Bekker M, Buckle C, Vink M, Seedat S, Bardien S, Carr J and Abrahams S (2020) Association Between a Variable Number Tandem Repeat Polymorphism Within the

DAT1 Gene and the Mesolimbic

Pathway in Parkinson's Disease.

Front. Neurol. 11:982.

doi: 10.3389/fneur.2020.00982
The loss of ventral striatal dopaminergic neurons in Parkinson's disease (PD) predicts an impact on the reward system. The ventrostriatal system is involved in motivational processing and its dysfunction may be related to non-motor symptoms such as depression and apathy. We previously documented that patients with PD had blunted Blood Oxygen Level Dependent functional magnetic resonance imaging (BOLD fMRI) reward task related activity during both reward anticipation (i.e., in the ventral striatum) and reward outcome (i.e., in the orbitofrontal cortex). Evidence for the modulation of brain function by dopaminergic genes in PD is limited. Genes implicated in dopamine transmission, such as the dopamine transporter gene (DAT1) may influence the clinical heterogeneity seen in PD, including reward processing. This study therefore sought to determine whether genetic differences in the DAT gene are associated with brain activity associated with response to reward in PD patients and controls. A sample of PD cases on treatment $(n=15)$ and non-PD controls $(n=30)$ from an ethnic group unique to South Africa were genotyped. We found a three-way interaction between GENOTYPE $\times$ BOLD $f M R I$ REWARD $\times$ DIAGNOSIS $\left[F_{(1,40)}=4.666, p=0.037\right.$, partial $\left.\eta^{2}=0.104\right]$. PD patients with the DAT1 homozygous $10 / 10$ repeat genotype showed a relative decrease in orbitofrontal cortex reward outcome related activity compared to the patient group who did not have this repeat. PD patients with other genotypes showed an expected increase in orbitofrontal cortex reward outcome related activity compared to controls. Given the small sample size of the PD group with the 10/10 repeat, these results should be considered preliminary. Nevertheless, these preliminary findings highlight the potential modulation of dopamine transporter polymorphisms on orbitofrontal reward system activity in PD and highlight the need for further studies.

Keywords: DAT1, SLC6A3, Parkinson's disease, monetary incentive delay, orbitofrontal cortex, ventral striatum, functional magnetic resonance imaging 


\section{INTRODUCTION}

Parkinson's disease (PD) is characterized by decreased dopaminergic availability in the brain, especially in the striatum (1). Changes in striatal dopaminergic tone in PD have been related to cognitive as well as reward processing abnormalities (2). Further, differences in fronto-striatal reward processing have been demonstrated in both medicated and unmedicated patients $(2,3)$. Specifically, blunted neural activity related to performance on a reward task was found to be a distinguishing factor in PD patients when compared to similarly aged controls (3). Brain activity patterns associated with reward may be linked to the non-motor symptoms of PD such as apathy (4) and impulsivity (5), and warrant investigation.

There is a growing body of literature documenting an association between candidate genes involved in the dopamine system and reward related brain activity (6-8). This includes the commonly occurring 10 -repeat allele of the $40 \mathrm{bp}$ variable number tandem repeat (VNTR) polymorphism within the Dopamine Active Transporter (DAT1) gene, also referred to as solute carrier family 6 member 3 (SLC6A3). Although the individual frequencies of the DAT1 repeats differ between ethnic groups, the 10-repeat allele is still the most common with frequencies ranging from 37 to $93 \%$ across several ethnic groups (9). The majority of in vitro studies have shown that the 10-repeat allele is associated with increased DAT1 expression in comparison to a commonly occurring 9repeat (10-12). Increased DAT1 expression leads to increased activity of DAT1 and increased dopamine uptake, which results in a decrease of dopamine levels in the synapse, and this could potentially be related to poorer reward related activity in the ventral-striatal system. Using single photon emission computed tomography (SPECT), subjects homozygous for the DAT 10-repeat allele had a $22 \%$ relative increase in DAT protein availability in homozygous DAT 10-repeat homozygotes compared to those with the 9-repeat/10-repeat genotype (10). Using Quantitative real-time Reverse Transcription Polymerase Chain Reaction (RT-PCR), it was shown that increased levels of DAT1 expression were associated with the number of 10repeat alleles (11). Radioligand binding and immunoblotting techniques also revealed statistically significant differences in DAT expression attributable to the DAT1 genotype, with lower the DAT1 density for the 9- and 10-repeat variants (12). Not all studies have found increased expression of DAT1 associated with this allele, with some reporting the opposite (13). Blood oxygen level dependent functional MRI (fMRI) nevertheless demonstrated relatively decreased reward anticipatory activity in the ventral striatum as well as decreased reward outcome related activity in the orbitofrontal cortex during a Monetary Incentive Delay (MID) reward processing task in individuals with the DAT1 10/10 repeat, compared to those with the $9 / 9$ repeat (6). The MID is known to robustly activate the ventral striatum during reward anticipation and orbitofrontal cortex during positive reward outcome (14). This finding was replicated in another reward processing study examining orbitofrontal and ventral striatal activity in DAT1 10/10-repeat vs. 9/9 repeat carriers, showing greater responses to smoking vs. non-smoking cues (15). However, there were contrasting findings in a study examining the impact of genotype on reward processing thought to underlie long-term memory formation where the DAT1 10-repeat homozygotes demonstrated increased striatal activity compared with 9/10-repeat heterozygotes (8).

As the relationship between DAT1 genotypes and reward processing is further clarified, it is important to examine the impact that the DAT1 genotype could have on the course of illnesses such as PD, where disease related alterations in dopamine tone are particularly evident (16). To our knowledge, there are no studies that have examined the relationship between the DAT1 genotype and abnormalities in brain activation associated with reward processing in PD.

Previously, we identified a relative decrease in both anticipatory activity seen in the ventral striatum and reward outcome related activity in the orbitofrontal cortex in PD (3). Here, we aim to investigate the potential modulating effects of the DAT1 10/10 genotype on these brain changes in a subsample of $15 \mathrm{PD}$ patients and 30 matched healthy controls, drawn from a larger cohort, who were genotyped and underwent fMRI whilst performing a monetary incentive delay task $(17,18)$. Given the aforementioned findings in the literature, we predicted that PD patients relative to controls, with the DAT1 10/10 repeat genotype, compared with DAT1 heterozygotes, would have the lowest levels of reward related activity in both the ventral striatum during anticipation and in the orbitofrontal cortex during reward outcome.

\section{MATERIALS AND METHODS}

The study participants form part of a larger cohort examining the genomic and environmental signatures that are common to PD, Posttraumatic Stress Disorder, Schizophrenia and metabolic syndrome (named the "Shared Roots" study, MRCRFA-UFSP-01-2013). The study has been approved by Health Research Ethics Committee (HREC N13/08/115) of Stellenbosch University, Tygerberg Hospital, Cape Town, South Africa, with annual renewal.

All participants were recruited from the same geographical region in Cape Town, South Africa, were unrelated and matched to socioeconomic status (lower to middle income status). All self-identified as "mixed ancestry" which refers to an ethnic population unique to South Africa and resulting from an admixture of individuals of African, European and Asian ancestral origins (19). This is the first published report on DAT1 genotypes in a South African Mixed Ancestry population. A diagnosis of PD was clinically confirmed by a neurologist according to MDS diagnostic criteria (20). A healthy (nonPD) control group was recruited and matched for ethnicity. Controls did not have current significant psychopathology or other significant confounding medical conditions.

\section{Clinical Assessments}

All participants received a full clinical examination. They were screened for any confounding psychopathology using the MiniInternational Neuropsychiatric Interview (MINI version 6.0.0). 
The Unified Parkinson's Rating Scale (UPDRS) (Version 3.0) was completed for the PD patients (21). Handedness was determined by the Edinburgh Handedness Inventory (22). All participants were asked to take their PD medication as normal, prior to scanning. All participants received a urine drug screen immediately before their MRI scan. Participants with severe head injury, confounding intra-cranial pathology, current severe psychopathology and/or drug abuse and other medical conditions that could confound behavioral as well as fMRI measures were excluded.

\section{Genotyping of 40 bp DAT1 VNTR Polymorphism}

Venous whole blood was collected from all study participants for the genetic analyses. Genomic DNA was isolated with the use of an in-house phenol/chloroform method prior to 2016 and a salting-out precipitation method (Gentra Puregene Blood Kit), for samples collected post 2016. Polymerase chain reaction (PCR) amplification was performed using primers DAT1 forward: 5' -ATGGGGGTCCTGGTATGTCT-3' and reverse: 5' GGCACGCACCTGAGAGAAAT-3'; that were designed using OligoAnalyzer (www.idtdna.com/oligoanalyzer), BLAST (www. ncbi.nlm.nih.gov/BLAST/) and primerBLAST (www.ncbi.nlm. nih.gov/tools/primer-blast/) for optimal binding to the region of interest. PCR was performed in $25 \mu \mathrm{l}$ reactions which contained $0.4 \mu \mathrm{M}$ DAT1 forward and reverse primers (Inqaba biotec $^{\mathrm{TM}}$, South Africa), $0.075 \mu \mathrm{M}$ of each dNTP, $0.25 \mathrm{U} \mathrm{GoTaq}^{\circledR}$ G2 Flexi DNA Polymerase, 1x Colorless GoTaq ${ }^{\circledR}$ Flexi Buffer, $1.5 \mathrm{mM} \mathrm{MgCl}_{2}$ solution (Promega, Madison, WI, USA) and 30 ng genomic DNA. The PCR conditions comprised of: initial denaturation at $95^{\circ} \mathrm{C}$ for $10 \mathrm{~min}$; followed by 35 cycles of denaturation at $93^{\circ} \mathrm{C}$ for $1 \mathrm{~min}$, annealing at $58^{\circ} \mathrm{C}$ for $30 \mathrm{~s}$ and extension at $72^{\circ} \mathrm{C}$ for $1 \mathrm{~min}$; and a final extension step at $72^{\circ} \mathrm{C}$ for $10 \mathrm{~min}$ using a thermocycler (Applied Biosystems, GeneAmp ${ }^{\circledR}$ PCR System 2700, Singapore). The PCR product was visualized using electrophoresis on a $1 \%$ SeaKem ${ }^{\circledR}$ LE Agarose gel (Consort Electrophoresis Power Supply, 800 Series, E844, Belgium). Genotyping was carried out by comparing the size of the PCR product, visualized on the agarose gel, to the expected product size determined based on the reference DNA sequence (NM 001044.5) in Ensembl (www.ensembl.org/index.html).

\section{Monetary Incentive Delay (MID) fMRI Paradigm}

All participants performed a modified version of the MID task (23). To enhance task comprehension, as well as keep the number of scan acquisitions to a minimum, only reward and neutral cues were used in this task. The task is described in detail elsewhere (23). Briefly, during each scan trial participants were required to respond as rapidly as possible when a target cue was presented. A smiling face immediately preceded the target, to indicate a potentially rewarding trial, and a neutral face was presented prior to neutral trials. After seeing the face cue, a blue star was shown for a short pseudo random interval immediately followed by the target cue (i.e., reward anticipation). If a participant responded in time to the target cue, a screen with green lettering appeared indicating the total reward won (i.e., reward outcome). If a participant did not respond in time, red letters appeared. During reward trials, the monetary reward was incrementally increased (fixed increments of ZAR10) (see Figure 1).

The reward anticipation period as well as the inter-trial interval were "jittered" to reduce collinearity between reward anticipation and reward outcome (mean duration 3,286 ms, range $779-6,729 \mathrm{~ms}$; mean duration $3,535 \mathrm{~ms}$, range 1,029 $6,979 \mathrm{~ms}$, respectively). The reward outcome period was $2,000 \mathrm{~ms}$ per trial. The entire task therefore consisted of 60 trials, with a mean duration of 9,571 ms (range 4,946-16,107 ms), resulting in a total task duration of 9 min $35 \mathrm{~s}$.

To ensure an equal number of rewarded and unrewarded trials, the duration of the target cue was adapted to the fastest response time of the participant during a training session. By matching task performance across subjects in this way, we controlled for differing levels of performance across the groups. The target score was set to approximately ZAR150 ( 10 USD) for each group.

\section{Behavioral Data Analysis}

Neutral correct trials and rewarded trials were compared between case-control (diagnostic) and DAT1 genotype groups using a repeated measures analysis of variance (RMANOVA), modeling for REWARD (i.e., neutral correct vs. rewarded trials) $\times$ DIAGNOSIS (i.e., PD and controls) $\times$ GENOTYPE (i.e., DAT1 $10 / 10$ repeat vs. other genotypes) interaction effects. Monetary reward across diagnostic and genotype groups was compared with a standard $t$-test. If the 3 -way modeling for REWARD $\times$ DIAGNOSIS $\times$ GENOTYPE interaction was significant, post-hoc testing was performed to identify whether the 3 -way interaction was driven by disease status or genotype.

\section{Image Acquisition}

Scans were acquired on a $3 \mathrm{~T}$ Siemens Allegra at the Combined Universities Brain Imaging Center (CUBIC). A total of 360 whole-brain 2D-EPI images $(\mathrm{TR}=1,600 \mathrm{~ms}$, $\mathrm{TE}=23 \mathrm{~ms}$, flip-angle: 72.5 degrees, FOV: $256 \times 256$, 30 slices, $4 \mathrm{~mm}$ isotropic voxels) were acquired in $9 \mathrm{~min} 35 \mathrm{~s}$. For image registration, a T1 ME-MPRAGE weighted structural scan was acquired $(\mathrm{TR}=2,530 \mathrm{~ms}$; TE1 $=1.53 \mathrm{~ms}$ TE2 $=3.21, \mathrm{~ms}$, TE3 $=4.89 \mathrm{~ms}$, $\mathrm{TE} 4=6.57 \mathrm{~ms}$, flip-angle: 7 degrees, FoV: $256 \mathrm{~mm}, 128$ slices, 1 isotropic voxel size) (24).

\section{Image Pre-processing}

Images were analyzed using SPM12 (http://www.fil.ion.ucl.ac.uk/ spm/software/spm12/). Pre-processing and first-level statistical analysis was undertaken as previously described (3). In brief, pre-processing involved correction for slice timing differences, re-alignment to correct for head motion, spatial normalization to the Montreal Neurological Institute template brain, and spatial smoothing to accommodate inter-individual differences in neuro-anatomy. Head motion parameters were analyzed to ensure that the maximum motion did not exceed a predefined threshold (scan-to-scan $>2 \mathrm{~mm}$ ). 


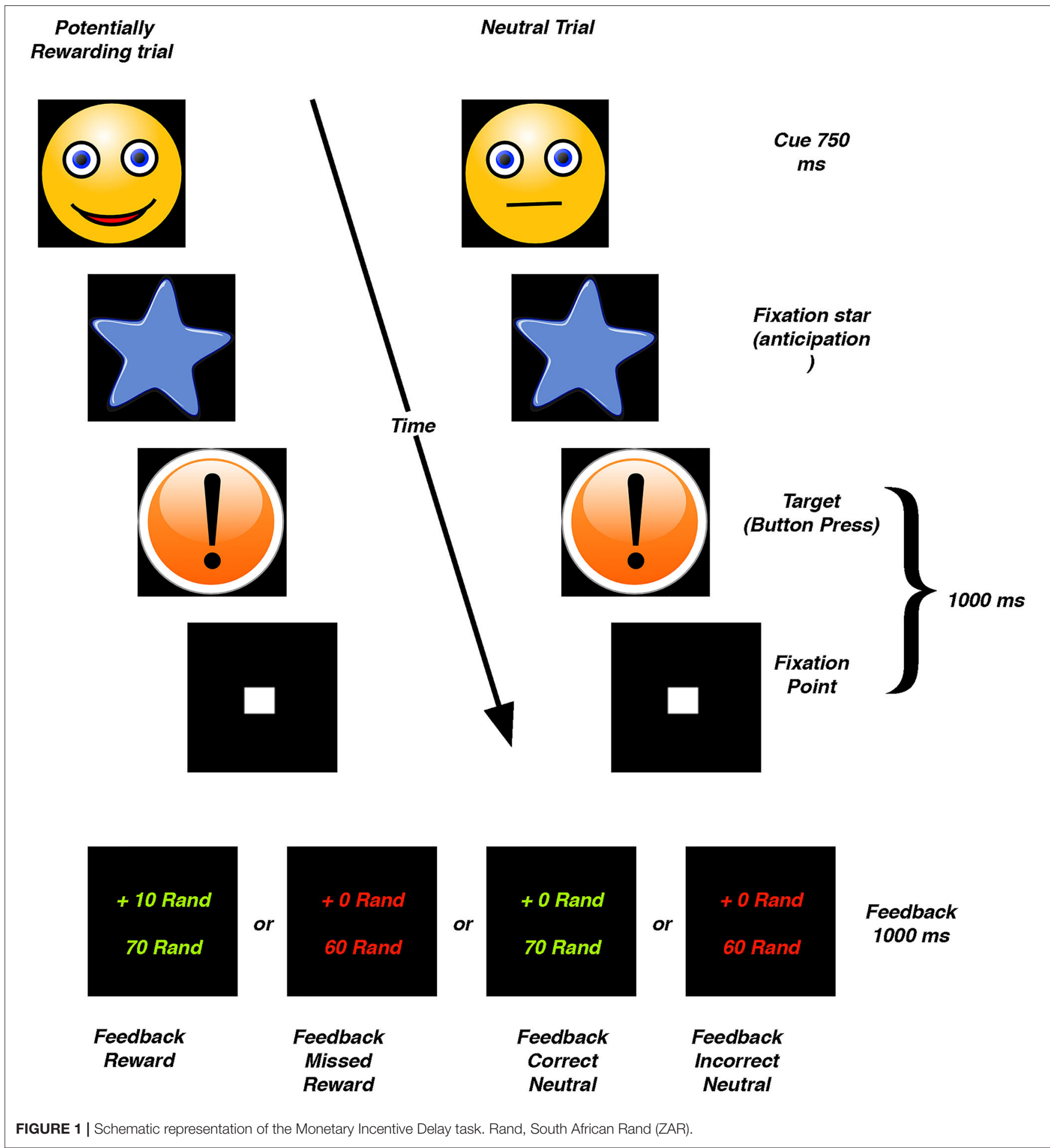

\section{First Level fMRI Statistical Analysis}

The pre-processed time-series data for each participant was analyzed using a standard general linear model (GLM) analysis. The model consisted of six factors of interest, representing haemodynamic changes time-locked to trial periods of (1) anticipation of receiving a potential reward, i.e., during and after the presentation of the reward cue (reward anticipation), (2) the lack of reward anticipation during and after a neutral cue (neutral anticipation), (3) feedback reflecting when money was received for a successful reward trial (reward outcome), (4) feedback when no reward was received, (5) feedback reflecting when the button was pressed in time during a neutral 
TABLE 1 | Demographics of the 45 study participants.

\begin{tabular}{|c|c|c|c|c|c|c|}
\hline & \multicolumn{2}{|c|}{$\begin{array}{l}\text { Patients } \\
n=15\end{array}$} & \multicolumn{2}{|c|}{$\begin{array}{c}\text { Controls } \\
n=30\end{array}$} & \multirow[b]{2}{*}{ Test score } & \multirow[b]{2}{*}{$p$-value } \\
\hline & Mean & SD & Mean & SD & & \\
\hline Age & 61.59 & 9.56 & 56.56 & 6.59 & $t=2.070$ & $0.04^{*}$ \\
\hline $\operatorname{Sex}(M / F)$ & $11 / 4$ & & $12 / 18$ & & $x^{2}=4.447$ & $0.04^{*}$ \\
\hline Handedness (R/L) & $14 / 1$ & & $29 / 1$ & & $X^{2}=0.262$ & 0.61 \\
\hline Months since diagnosis & 56.6 & 41.92 & & & & \\
\hline LED (mg/day) & 560 & 307.76 & & & & \\
\hline Hoehn \& Yahr staging & 2.57 & & & & & \\
\hline ADL (best/worst) & 73.33/65.33 & & & & & \\
\hline Reward Won (ZAR) & 118.67 & & 121.67 & & $t=-0.309$ & 0.76 \\
\hline \multicolumn{7}{|l|}{ fMRI motion parameters } \\
\hline Mean motion & 0.09 & 0.03 & 0.10 & 0.03 & $t=-1.141$ & 0.26 \\
\hline Maximum motion & 0.47 & 0.28 & 0.52 & 0.31 & $t=-0.517$ & 0.61 \\
\hline Total number of movements & 124.87 & 80.20 & 138.27 & 64.75 & $t=-0.604$ & 0.55 \\
\hline Mean rotation & 0.001 & 0.0003 & 0.001 & 0.0004 & $t=-0.867$ & 0.39 \\
\hline Maximum rotation & 0.006 & 0.0025 & 0.008 & 0.0070 & $t=-0.803$ & 0.43 \\
\hline
\end{tabular}

*Significant at $p<0.05$ level.

trial (neutral correct outcome), and (6) feedback reflecting an incorrect response in a neutral trial, i.e., when the target was missed when no reward was offered (Figure 1). The onset of the factors modeling anticipation (duration range 1,529$7,479 \mathrm{~ms}$ ) was at the presentation of the cue, while the onset of the factors modeling feedback (duration: 2,000 ms) was at the presentation of the target, including the button press to the target and subsequent feedback (see Figure 1). Motion parameters from the realignment procedure were included as factors of no interest. Low frequency drifts were removed from the signal by applying a high-pass filter with a cut-off frequency of $128 \mathrm{~Hz}$.

\section{Region of Interest Analyses}

Primary analyses were performed in one region of interest (ROI): the combined bilateral ventral striatum for anticipation, and combined bilateral orbitofrontal cortex for reward outcome, based on previous findings (23). These regions were defined using the Automated Anatomical Labeling (AAL)-atlas (25) and the Oxford-GSK-Imanova Striatal Connectivity Atlas for the ventral striatum (26). For each participant, the mean activation level (expressed as percent signal change) during the contrasts of interest specific to reward anticipation and reward outcome (reward anticipation, neutral anticipation, reward outcome, and neutral correct outcome) was averaged over all the voxels of each ROI using SPM12 and custom MATLAB R2019a scripts.

Similar to the behavioral data analysis, these values were used in a RMANOVA, testing for main and group effects in activation levels between neutral vs. potentially rewarding trials, reward anticipation vs. reward outcome, and correct neutral trials vs. positive reward outcome. As in the behavioral analysis, we modeled for a REWARD $\times$ DIAGNOSIS $\times$ GENOTYPE interaction effect.

\section{RESULTS}

The Shared Roots cohort comprised 81 PD patients and 79 controls. All participants were genotyped for the 40 bp DAT1 VNTR. Data was originally collected from 2 separate scan sites. Due to the low number of controls relative to patients available at the second site ( $n=6$ with DAT1 $10 / 10$ genotype, $n=2$ with other genotypes) which resulted in an unbalanced sample for the second scan site, we chose only to include data from the first scan site. Of these, 18 patients and 39 controls had fMRI, T1 structural scan and genotype data. Three PD patients and seven controls were excluded due to the presence of motion or other scanner related artifacts. Two controls were excluded due to poor task performance. This resulted in a final sample of 15 patients and 30 controls. The demographics of the 45 study participants are reflected in Table 1. There was a small but significant difference in age between the cases and controls. We therefore included age as a covariate in all analyses. Although there were significantly fewer females present in the patient group than in the control group, we chose not to correct for this in our final model, as we found no sex-based differences on the MID in our larger sample $(3,18)$. Patient and control groups were also matched in terms of several important motion parameters (Table 1) (27).

Similar to European cohorts, the DAT1 10/10 repeat was the most commonly occurring repeat in the sample $(45.8 \%)$, followed by the $10 / 9$ repeat. Interestingly, our sample contained few DAT1 9/9 repeats (4.2\%, see Figure 2) unlike cohorts of European ancestry where it is often frequent. Importantly, there was no significant difference in the frequencies of the genotype subgroups, which were balanced between patients and controls $\left(\mathrm{X}^{2}=2.179, p=0.14\right)$. As we had an a priori hypothesis for the DAT1 10/10 repeat and had observed a relatively low frequency of the other repeats, we divided the sample into two groups: a "10/10 repeat" genotype group compared to all the other genotypes. 


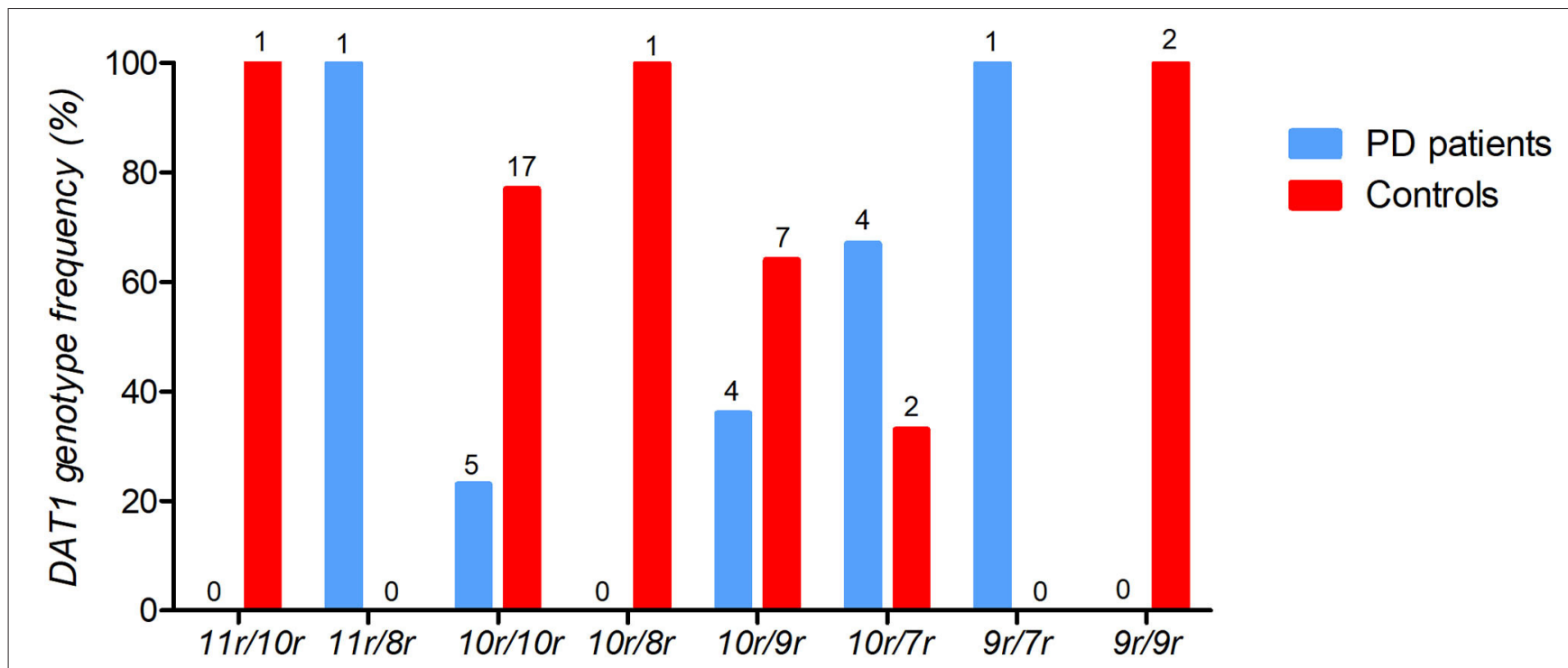

FIGURE 2 | The frequencies of DAT1 variable number tandem repeat (VNTR) for PD patients $(n=15)$ and healthy controls $(n=30)$. The percentage of DAT1 VNTR frequencies are depicted with the individual counts $(n)$ displayed above each genotype bar.

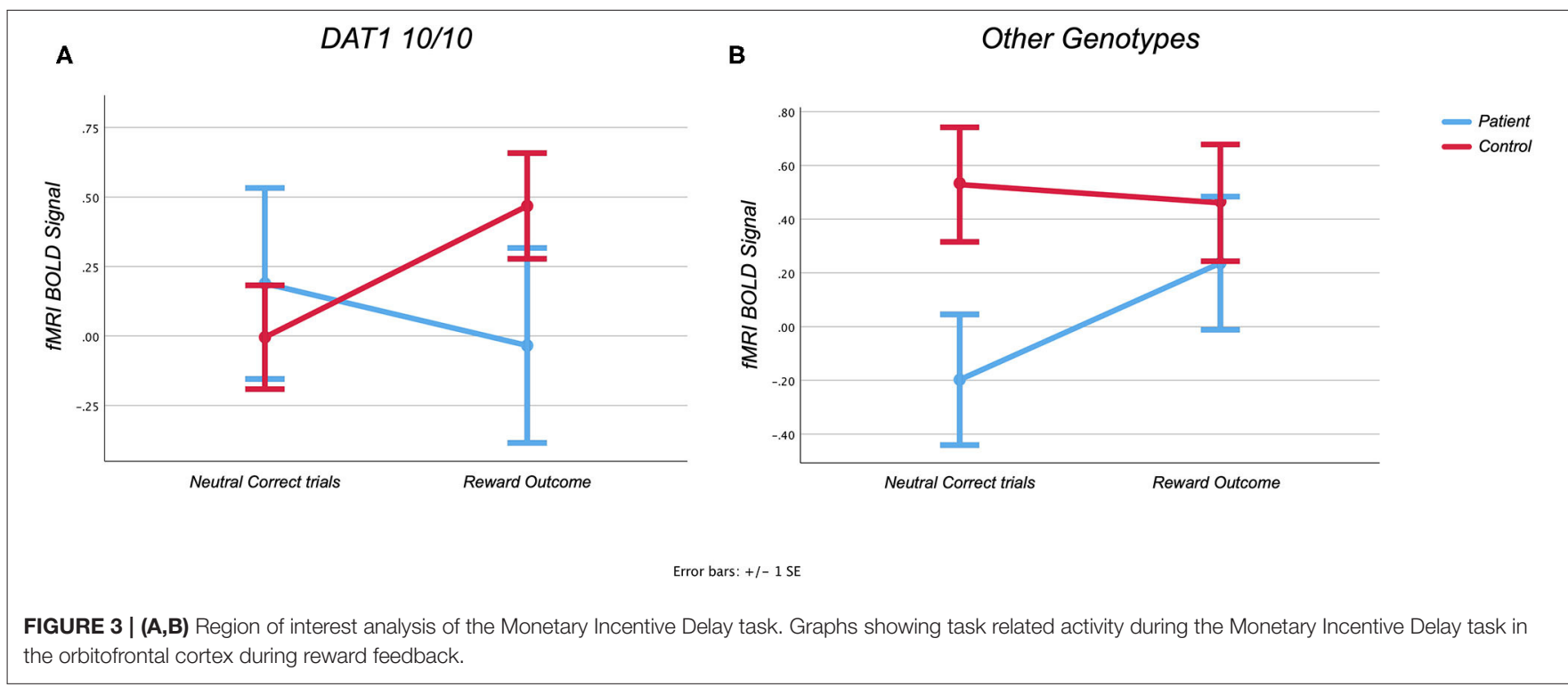

\section{No Difference Observed in Behavioral Data}

Since the task was adjusted according to participant's performance level, patients and controls received an equal amount of reward $\left[t_{(43)}=-0.309, p=0.759\right]$. Both patients and controls appropriately responded more rapidly to rewarded trials $\left[F_{(1,41)}=5.633, p=0.022\right]$, but there was no REWARD $\times$ GENOTYPE interaction effect $\left[F_{(1,41)}=0.103, p=0.749\right]$.

\section{No Reward Anticipation Effect Observed in the Ventral Striatum}

Contrary to our hypothesis, we found no main effect for reward anticipation in the ventral striatum $\left[F_{(1,41)}=1.615, p=0.211\right]$, nor a DIAGNOSIS interaction effect $\left[F_{(1,41)}=2.227, p=0.143\right]$.

\section{Reward Outcome Effect Observed in the Orbitofrontal Cortex}

Although there was no main effect for reward outcome in the orbitofrontal cortex $\left[F_{(1)}=1.172, p=0.285\right]$, there was a three-way REWARD $\times$ DIAGNOSIS $\times$ GENOTYPE interaction effect $\left[F_{(1,40)}=4.666, p=0.037\right]$, while controlling for age, in line with our hypothesis (see Figure 3). As predicted, after post-hoc testing, Parkinson's patients who have the DAT1 10/10 genotype demonstrated a decrease on average in bold signal from neutral to rewarded trials, whereas those with other genotypes demonstrated a normal increase in activity $\left[F_{(1)}=1.678, p=\right.$ 0.22 , partial $\left.\eta^{2}=0.123\right)$. Interestingly, controls demonstrate the opposite effect, showing a relative increase in those that 
have the DAT1 10/10 repeat, with an absent response for the other genotypes $\left[F_{(1)}=3.017, p=0.094\right.$, partial $\left.\eta^{2}=0.101\right]$. Uncorrected post-hoc testing however failed to yield significant results. A marginally larger effect was noted when comparing patients with and without the DAT1 10/10 genotype (partial $\eta^{2}$ $=0.123$ ), than when comparing controls with and without the genotype (partial $\eta^{2}=0.101$ ). This suggests that the three-way interaction effect in our main analysis was driven more by the patients with and without the DAT1 10/10 genotype relationship. As post-hoc testing could not adequately distinguish between the various subgroups and given the small sample size, particularly that of the Parkinson's group with the DAT1 10/10 repeat $(n=5)$, these results should be considered exploratory.

The blunted response in the control group which did not have the DAT1 10/10 repeat can still be expected for this age group. Results remained unchanged, however, when the age covariate was removed. A sample using a wider age range would be needed to adequately address this question.

\section{Exploratory Whole Brain Voxel Wise Analysis}

As we had a specific hypothesis, directed at specific brain regions we did not focus on whole brain analyses. Whole brain exploratory analysis did not yield any additional information.

\section{DISCUSSION}

We investigated the potential genetic underpinnings of reward processing abnormalities seen in PD compared to healthy controls. As predicted, we found evidence for poorer reward outcome-based reactivity in the orbitofrontal cortex for patients with the DAT1 10/10 genotype compared to those with other genotypes. Contrary to our hypothesis, we found no such differences in the ventral striatum during reward anticipation. To our knowledge this is the first-time reward-related functional genotypes have been investigated in PD.

Our finding that DAT1 10/10 carriers had abnormal reward outcome related activity in the orbitofrontal cortex could reflect an increased vulnerability for PD-related dopaminergic cell loss in this region. Indeed, it has been shown that the DAT1 10/10 genotype is associated with lower synaptic dopamine availability due to possible increased levels of dopamine transporters (10). Not all studies reported this however, with some suggesting the opposite (13). Our results corroborate a potential hypodopaminergic state in the DAT1 10/10 group, as BOLD fMRI activity has been found to correlate with dopamine reactivity in this region (14). However, further exploration of this link with more direct methods such as the use of positron emission tomography (PET) would be needed to confirm this. Although the DAT1 10/10 genotype is one of the more common functional variants reported in the literature, it is possible that there are other unexamined variants that are stronger predictors of dopaminergic hypofunction. Our results confirm that DAT1 $10 / 10$ is a potential predictor of reward function variability in diseased states. As our present sample only includes patients on treatment, we cannot account for potential treatment effects.
It could be that PD medication has an impact on the normal dopaminergic tone of the ventral tegmental area, which could also have a differential impact across genotypes. As our patients were not assessed while medication free and dopamine activity was not directly measured, we cannot comment on treatment effects. Further treatment effect studies are advised. Interestingly, although Parkinson's patients without the 10/10 DAT1 repeat showed a normal increase in reward outcome related activity, controls without the 10/10 repeat showed a relatively flattened out response. The absence of a response in the controls could be age related, as a similar flattened out response has been observed in similarly aged healthy controls in previous studies $(3,18)$. The relative increase in reward outcome related activity Parkinson's patients without the DAT 1 10/10 repeat could possibly reflect treatment effects. Again, future studies investigating medication and genotype interaction effects in larger number of patients and controls are needed to substantiate this finding.

Contrary to our previous findings, we did not find any reward anticipatory related activity in the ventral striatum in this particular sub-sample, nor any effect of genotype in this region for this subgroup. The absence of a reward anticipation effect could be explained by poor data quality or poor task comprehension. This was unlikely to be the case in our study as our groups also showed low levels of motion and did not differ on important measures of motion. All groups also demonstrated good task comprehension, as they increased their response times appropriately during rewarded trials. Another potential explanation is the relative older age of the current subsample, which could explain the general lack of signal for this region. Indeed, it has been found that ventral striatal but not orbitofrontal activity per se, decreased with normal aging (18).

Although our findings do indeed substantiate our hypothesis that reward related functioning is related, at least in part, to DAT1 genotype, our sample is small, and therefore these findings should be considered as exploratory. Although we found significant differences in the orbitofrontal regions, we cannot completely rule out similar findings for the ventral striatum due to our limited sample size. Furthermore, although our study supports functional associations with the commonly occurring DAT1 genotype, this does not necessarily mean that it is the only or even the best predictor of reward related functioning. Future, larger studies should also explore other dopamine-related genes such as catechol-O-methyltransferase (COMT) (6).

Despite these limitations, our study has important implications. Reward related function loss, and PD nonmotor symptoms by extension, could be exacerbated in certain vulnerable genotypes. This should be considered in future studies of genetic vulnerability and treatment in PD. Genetic risk factors could potentially play an important role in the non-motor symptoms of PD.

\section{DATA AVAILABILITY STATEMENT}

The raw data supporting the conclusions of this article will be made available by the authors, without undue reservation, to any qualified researcher. 


\section{ETHICS STATEMENT}

The studies involving human participants were reviewed and approved by Health Research Ethics Committee (HREC N13/08/115) of Stellenbosch University, Tygerberg Hospital, Cape Town, South Africa. The patients/participants provided their written informed consent to participate in this study.

\section{AUTHOR CONTRIBUTIONS}

SP: study design, fMRI task administration supervision, data processing, analysis, interpretation, and lead author on manuscript preparation. MB: genetic samples analysis, interpretation, and manuscript preparation. $\mathrm{CB}$ and MV: fMRI data processing, analysis, and interpretation. SS, SB, and JC: study design, interpretation, and manuscript preparation.

\section{REFERENCES}

1. Tedroff J, Pedersen M, Aquilonius SM, Hartvig P, Jacobsson G, Långström B. Levodopa-induced changes in synaptic dopamine in patients with Parkinson's disease as measured by [11C]raclopride displacement and PET. Neurology. (1996) 46:1430-6. doi: 10.1212/WNL.46.5.1430

2. Schott BH, Niehaus L, Wittmann BC, Schütze H, Seidenbecher CI, Heinze $\mathrm{H}-\mathrm{J}$, et al. Ageing and early-stage Parkinson's disease affect separable neural mechanisms of mesolimbic reward processing. Brain. (2007) 130:2412-24. doi: 10.1093/brain/awm147

3. Plessis du S, Bossert M, Vink M, van den Heuvel L, Bardien S, Emsley R, et al. Reward processing dysfunction in ventral striatum and orbitofrontal cortex in Parkinson's disease. Parkinsonism Relat Disord. (2017) 48:82-8. doi: 10.1016/j.parkreldis.2017.12.024

4. Chong TTJ, Bonnelle V, Manohar S, Veromann K-R, Muhammed K, Tofaris GK, et al. Dopamine enhances willingness to exert effort for reward in Parkinson's disease. Cortex. (2015) 69:40-6. doi: 10.1016/j.cortex.2015.04.003

5. Dagher A, Robbins TW. Personality, addiction, dopamine: insights from Parkinson's disease. Neuron. (2009) 61:502-10. doi: 10.1016/j.neuron.2009.01.031

6. Dreher J-C, Kohn P, Kolachana B, Weinberger DR, Berman KF. Variation in dopamine genes influences responsivity of the human reward system. Proc Natl Acad Sci USA. (2009) 106:617-22. doi: 10.1073/pnas.0805517106

7. Dubol M, Trichard C, Leroy C, Sandu A-L, Rahim M, Granger B, et al. Dopamine transporter and reward anticipation in a dimensional perspective: a multimodal brain imaging study. Neuropsychopharmacology. (2018) 43:820-7. doi: 10.1038/npp.2017.183

8. Wittmann BC, Tan GC, Lisman JE, Dolan RJ, Düzel E. DAT genotype modulates striatal processing and long-term memory for items associated with reward and punishment. Neuropsychologia. (2013) 51:2184-93. doi: 10.1016/j.neuropsychologia.2013.07.018

9. Kang AM, Palmatier MA, Kidd KK. Global variation of a 40-bp VNTR in the 3'-untranslated region of the dopamine transporter gene (SLC6A3). BPS. (1999) 46:151-60. doi: 10.1016/S0006-3223(99)0 0101-8

10. Heinz A, Goldman D, Jones DW, Palmour R, Hommer D, Gorey JG, et al. Genotype influences in vivo dopamine transporter availability in human striatum. Neuropsychopharmacology. (2000) 22:133-9. doi: 10.1016/S0893-133X(99)00099-8

11. Mill J, Asherson P, Browes C, D'Souza U, Craig I. Expression of the dopamine transporter gene is regulated by the 3' UTR VNTR: evidence from brain and lymphocytes using quantitative RT-PCR. Am J Med Genet. (2002) 114:975-9. doi: 10.1002/ajmg.b.10948
SA: genetic sample analysis and interpretation. All authors contributed to the article and approved the submitted version.

\section{FUNDING}

The authors were financially supported by the National Research Foundation of South Africa (Grant Number: 106052), the South African Medical Research Council (Self-Initiated Research Grant), and Stellenbosch University. Funding was also received from a South African Medical Research Council Flagship Grant (RFA-UFSP-01-2013). We also acknowledge the support of the NRF-DST Center of Excellence for Biomedical Tuberculosis Research; South African Medical Research Council Center for Tuberculosis Research; Division of Molecular Biology and Human Genetics, Faculty of Medicine and Health Sciences, Stellenbosch University, Cape Town.

12. VanNess SH, Owens MJ, Kilts CD. The variable number of tandem repeats element in DAT1 regulates in vitro dopamine transporter density. BMC Genet. (2005) 6:1-11. doi: 10.1186/1471-2156-6-55

13. van Dyck CH, Malison RT, Jacobsen LK, Seibyl JP, Staley JK, Laruelle M, et al. Increased dopamine transporter availability associated with the 9-repeat allele of the SLC6A3 gene. J Nucl Med. (2005) 46:745-51.

14. Knutson B, Gibbs SEB. Linking nucleus accumbens dopamine and blood oxygenation. Psychopharmacology. (2007) 191:813-22. doi: 10.1007/s00213-006-0686-7

15. Franklin TR, Lohoff FW, Wang Z, Sciortino N, Harper D, Li Y, et al. DAT genotype modulates brain and behavioral responses elicited by cigarette cues. Neuropsychopharmacology. (2009) 34:717-28. doi: 10.1038/npp.2 008.124

16. Morrish PK, Sawle GV, Brooks DJ. Clinical and [18F] dopa PET findings in early Parkinson's disease. J Neurol Neurosurg Psychiatr. (1995) 59:597-600. doi: 10.1136/jnnp.59.6.597

17. Knutson B, Fong GW, Adams CM, Varner JL, Hommer D. Dissociation of reward anticipation and outcome with event-related fMRI. Neuroreport. (2001) 12:3683-7. doi: 10.1097/00001756-20011204000016

18. Vink M, Kleerekooper I, van den Wildenberg WPM, Kahn RS. Impact of aging on frontostriatal reward processing. Hum. Brain Mapp. (2015) 36:2305-17. doi: 10.1002/hbm.22771

19. Patterson N, Petersen DC, van der Ross RE, Sudoyo H, Glashoff RH, Marzuki $\mathrm{S}$, et al. Genetic structure of a unique admixed population: implications for medical research. Hum Mol Genet. (2010) 19:411-9. doi: 10.1093/hmg/d dp505

20. Postuma RB, Berg D, Stern M, Poewe W, Olanow CW, Oertel W, et al. MDS clinical diagnostic criteria for Parkinson's disease. Mov Disord. (2015) 30:1591-601. doi: 10.1002/mds.26424

21. Goetz CG, Fahn S, Martinez-Martin P, Poewe W, Sampaio C, Stebbins GT, et al. Movement disorder society-sponsored revision of the unified parkinson's disease rating scale (MDS-UPDRS): process, format, and clinimetric testing plan. Mov Disord. (2007) 22:41-7. doi: 10.1002/mds.21198

22. Oldfield RC. The assessment and analysis of handedness: the Edinburgh inventory. Neuropsychologia. (1971) 9:97-113. doi: 10.1016/0028-3932(71)90067-4

23. Knutson B, Adams CM, Fong GW, Hommer D. Anticipation of increasing monetary reward selectively recruits nucleus accumbens. J Neurosci. (2001) 21:RC159. doi: 10.1523/JNEUROSCI.21-16-j0002.2001

24. van der Kouwe AJW, Benner T, Salat DH, Fischl B. Brain morphometry with multiecho MPRAGE. NeuroImage. (2008) 40:559-69. doi: 10.1016/j.neuroimage.2007.12.025 
25. Tzourio-Mazoyer N, Landeau B, Papathanassiou D, Crivello F, Etard $\mathrm{O}$, Delcroix $\mathrm{N}$, et al. Automated anatomical labeling of activations in SPM using a macroscopic anatomical parcellation of the MNI MRI single-subject brain. NeuroImage. (2002) 15:273-89. doi: 10.1006/nimg.20 01.0978

26. Tziortzi AC, Haber SN, Searle GE, Tsoumpas C, Long CJ, Shotbolt $\mathrm{P}$, et al. Connectivity-based functional analysis of dopamine release in the striatum using diffusion-weighted MRI and positron emission tomography. Cereb Cortex. (2014) 24:1165-77. doi: 10.1093/cercor/b hs397

27. Van Dijk KRA, Sabuncu MR, Buckner RL. The influence of head motion on intrinsic functional connectivity MRI. NeuroImage. (2012) 59:431-8. doi: 10.1016/j.neuroimage.2011.07.044
Conflict of Interest: The authors declare that the research was conducted in the absence of any commercial or financial relationships that could be construed as a potential conflict of interest.

The handling editor declared a past collaboration with the authors.

Copyright (c) 2020 du Plessis, Bekker, Buckle, Vink, Seedat, Bardien, Carr and Abrahams. This is an open-access article distributed under the terms of the Creative Commons Attribution License (CC BY). The use, distribution or reproduction in other forums is permitted, provided the original author(s) and the copyright owner(s) are credited and that the original publication in this journal is cited, in accordance with accepted academic practice. No use, distribution or reproduction is permitted which does not comply with these terms. 